\title{
Developments of multi-channel silicon avalanche photodiode sensors for low light imaging detection
}

\author{
H. Y. Lee', Jin-A Jeon, H. S. Lee, M. H. Lee \\ Center for Underground Physics, Institute for Basic Science (IBS) \\ 55 Expo-ro, Yuseong-gu, Daejeon 34126, Republic of Korea \\ E-mail: hylnate at nate.com
}

\section{J. Jegal, Hongjoo Kim}

Department of Physics, Kyungpook National University

80 Daehak-ro, Buk-gu, Daegu 41566 Republic of Korea

\begin{abstract}
Silicon avalanche photodiode (APD) is a highly sensitive semiconductor photo sensor that uses the photoelectric effect converting light to electric charge. APD has high a gain through avalanche multiplication in its p-n junction. APD also has various preferable characteristics such as high quantum efficiency, large dynamic range, light-weight form-factor, robustness and insensitivity to magnetic fields as well as low light measurement capability thanks to its gain of $\sim 100$. As a monolithic device, however, it usually has size of $\sim 5 \times 5 \mathrm{~mm}^{2}$. The APD sensor has appealed to various fields of experimental physics due to its low light detection. But its usage is still limited because of its small surface area. The photo sensor in an experiment usually is required to cover a large area. In this study, the p$\mathrm{n}$ junction of an APD was simulated with a sufficiently strong electric field enabling avalanche breakdown at $\sim 400 \mathrm{~V}$. We have designed large area APD sensors with channels of $5 \times 5$ and $10 \times 10$ to cover a wide area of $\sim 5 \times 5 \mathrm{~cm}^{2}$. The sensors were fabricated on a 6-inch $\mathrm{n}$-type silicon wafer in about 140 steps. We present the simulation, design and fabrication result for multi-channel silicon APD sensors manufactured in Korea.
\end{abstract}

The 39th International Conference on High Energy Physics (ICHEP2018)

4-11 July, 2018

Seoul, Korea

\section{${ }^{1}$ Speaker




\section{Introduction}

The APD has many advantages such as high speed, large dynamic range, compactness, robustness, and high quantum efficiency. The best advantage of the sensor is having an internal gain. The sensor has the same photocurrent generation mechanism as that of a general photodiode (PD), but the APD has high internal gain (> 1000) compared to the PD with only unit gain [1]. The sensor is being used as a promising light sensor in various physics experiments to detect low intensity light. At low temperatures, it can even measure single photon with low dark current [2]. We can, therefore, replace conventional Photo Multiplier Tube (PMT) with APD. However, it is usually used as a monolithic device with a limit in large area detection. We have simulated, designed and fabricated multi-channel APD sensors to cover the large area.

\section{Development of silicon APD sensor}

We have designed silicon APD sensors with arrays of pixels in an area of $5 \times 5 \mathrm{~cm}^{2}$ in order to replace 3 -inch PMT. Fig. 1 shows a sensor design with $10 \times 10$ array of pixels $\left(5 \times 5 \mathrm{~mm}^{2}\right)$ and $5 \times 5$ array of pixels $\left(10 \times 10 \mathrm{~mm}^{2}\right)$ in the same area. The sensors were simulated and fabricated with high electric fields enabling avalanche breakdowns at $\sim 400 \mathrm{~V}$ on a 6 -inch high resistivity wafer. The produced sensors have avalanche breakdown voltage of $\sim 400 \mathrm{~V}$ as designed. Fig. 2 shows the sensor gain of $\sim 100$ at an operating voltage of $380 \mathrm{~V}$. But the high level of dark current is also shown in the graph.

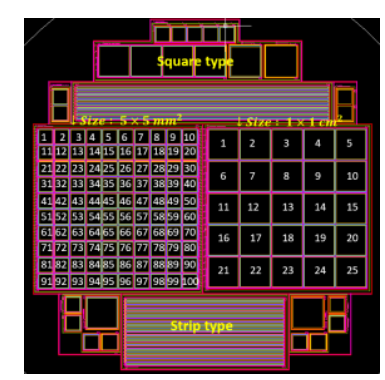

Fig. 1. Design of APDs with $5 \times 5$ and $10 \times 10$ arrays in the area of $5 \times 5 \mathrm{~cm}^{2}$.

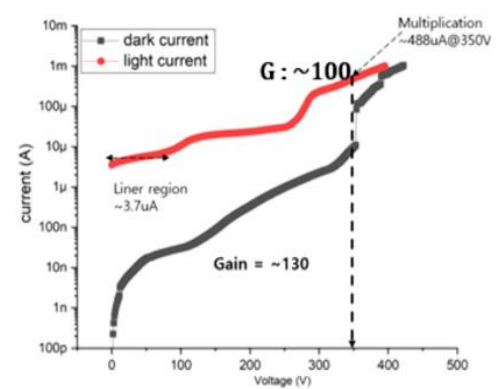

Fig. 2. Dark and light currents characteristics measured with an APD manufactured.

\section{Summary}

APD array sensors are developed to replace a conventional 3-inch PMT for a near future experiment. The silicon APD sensor can be used as a photo sensor with low noise characteristics at low temperature (i.e., $\mathrm{T}=100 \mathrm{~K}$ ). We have simulated, designed and fabricated array APD sensors. A fabricated prototype sensor has a breakdown voltage at about $400 \mathrm{~V}$. The Gain measured in a light test at wavelength of $600 \mathrm{~nm}$ is about 100 at room temperature. But the sensors found to have high leakage current levels in their operating ranges. In the next sensor development, we will improve the dark current level of the sensors.

\section{References}

[1] K. Dieters, et al., Investigation of the avalanche photodiodes for the CMS electromagnetic calorimeter operated at high gain, NIM A 461 (2001) 574.

[2] A. Dorokhov et al., Study of the Hamamatsu avalanche photodiode at liquid nitrogen temperatures, NIM A 504 (2003) 58. 